\title{
APUNTES SOBRE EL TESORO DEL BRONCE FINAL LLAMADO DE VALDEOBISPO
}

\author{
POR \\ JUAN JAVIER ENRIQUEZ NAVASCUES (*)
}

RESUMEN El tesorillo que se ha denominado de Valdeobispo (Cáceres, Extremadura) está constituido por un collar y cuatro brazaletes de oro macizo, cuya tipología lo relaciona con la orfebrería del Bronce final caracterizada por el influjo de las corrientes metalúrgicas atlánticas. La pieza más interesante es el collar, muy parecido a los de los tesoros de Berzocana y Baioes.

ABSTRACT The Valdeobispo hoard (Cáceres, Extremadura) consists of one necklace and four solid gold-bracelets, whose typology is related to the final Bronze Age goldwork characterized by the influence of Atlantic metallurgy.

The most interesting piece is the necklace, which is very similar to the ones from the Berzocana and Baioes' hoards.

Palabras clave Orfebrería. Bronce final atlántico. Extremadura.

Key words Goldwork. Atlantic Final Bronze Age. Extremadura.

No hace demasiado tiempo que se hizo tristemente famoso un tesorillo de joyas de oro pertenecientes a la Edad del Bronce, supuestamente encontrado en el término municipal de Valdeobipso (Cáceres). Hoy todavía colea el asunto de la desaparición de las piezas, denunciada por sus propietarios, y acciones judiciales dictadas para tratar de esclarecer los hechos continúan su curso. A pesar de ello, parece muy difícil que las piezas puedan ser recuperadas y por este motivo queremos dar a conocer los datos que sobre ellas tenemos, con una pequeña valoración de su importancia e interés como documento histórico y cultural.

Pero antes de centrar nuestra atención en el llamado tesoro de Valdeobispo, hay que dejar constancia del esfuerzo realizado por diversas personas para que las piezas pudieran pasar a ser custodiadas en un centro museológico con la mayor rapidez posible. De manera muy especial D. José M. Alvarez y D. Guillermo Kurtz, directores de los museos de Mérida y Badajoz, quienes colaboraron con nosotros en el intento de que la administración fuera ágil y adquiriese enseguida el tesorillo. Porque el caso fue que después de diversas negociaciones con intermediarios — se sabía de

(`) Consejería de Educación y Cultura. Junta de Extremadura. 
su existencia por haber sido of recido a distintos compradores potenciales-, los propietarios accedieron a depositar temporalmente las piezas en la Consejeria de Educación y Cultura de la Junta de Extremadura para su análisis, cumpliendo así, aunque sólo de forma parcial, lo establecido en la Ley del Patrimonio Histórico Español sobre hallazgos arqueológicos casuales (art. 44). Enseguida a las piezas se les incohó expediente para su declaración como Bienes de Interés Cultural (12-11-29, D.O.E. 16-11-89), pero tras unos meses se reintegraron a sus propietarios ante la perplejidad de más de uno. Una vez devueltas, sus propietarios las sacaron a subasta en una casa de Madrid con inusitada rapidez. La subasta, que levantó gran polémica, fue suspendida por la Consejería de Educación y Cultura de la Junta de Extremadura por no haber sido comunicada a tiempo tal y como marca la legislación vigente para los bienes con expediente abierto de B.I.C. Se trataba evidentemente de ganar tiempo. Más tarde, y previa comunicación, las piezas salieron a subasta pública en 11 millones de pts. sin que nadie pujara por ellas puesto que la citada Consejería hizo saber que las piezas iban a ser declaradas de interés social para su expropiación forzosa. Por fin, con fecha 14-III-89 (D.O.E. 21-III-89) se declaró el tesoro de Valdeobispo como Bien de Interés Social para expropiación forzosa. Curiosamente, muy poco antes de salir la resolución publicada, los propietarios denunciaban en una comisaria de Plasencia el robo del tesorillo en cuestión.

Hoy, como decíamos antes, la investigación judicial sigue abierta y para los propietarios se han dictado sanciones, que lógicamente han sido recurridas por ellos. La historia de la desaparición sigue pues sin resolverse y las diligencias continuan su curso, aunque las piezas ya es muy difícil que puedan ser controladas.

Hasta aqui los hechos escuetos, ya que explicaciones del por qué se reintegraron a sus supuestos descubridores ni a nosotros se nos han dado.

\section{PROCEDENCIA Y CIRCUNSTANCIAS DEL HALLAZgO}

Ciñéndonos al tesorillo, sus poseedores declararon que se trataba de un hallazgo casual ocurrido hacia años en el paraje Cruz de Clemente o Albanizo, situado en el término municipal de Valdeobipso. Otros testimonios sin embargo contradecían estas afirmaciones y aseguraban que no procedía de Valdeobispo aunque sí de Extremadura.

La actitud de los dueños y esta serie de testimonios desinteresados, que bajo la promesa de no mencionar nombres nos hicieron saber distintas personas sin vinculación aparente entre sí, provocó la duda. Ella aumentó cuando se supo la vinculación de los poseedores con negocios de joyería y los frecuentes viajes que realizaban por diversos pueblos extremeños para compraventa de oro y joyas. A pesar de todo se realizaron prospecciones en la finca Albanizo - y no excavaciones como dijeron algunos medios de comunicación- con resultados negativos, lo que en principio tampoco era de extrañar habida cuenta de los lugares y circunstancias en que han hecho su aparición piezas semejantes. El yacimiento del Bronce final más cercano a Valdeobispo que conocemos es el de Valcorchero, junto a Plasencia (Almagro Gorbea, 1977: 82 y ss.).

Por tanto, de las circunstancias del hallazo no sabemos a ciencia cierta nada. Ellos dijeron que fue arando, otros que fueron compradas lejos del pueblo. En cuanto al lugar, hay indicios bastante razonables para poner en tela de juicio cuanto menos a Valdeobispo y la finca Albanizo en concreto. Todos los testimonios coinciden sin embargo en que se trataba de objetos encontrados dentro de Extremadura.

\section{DESCRIPCION DE LAS PIEZAS (Fig. 1, Lám. IA)}

El tesorillo estaba formado por cinco objetos de oro macizo, un torques y cuatro brazaletes, con 

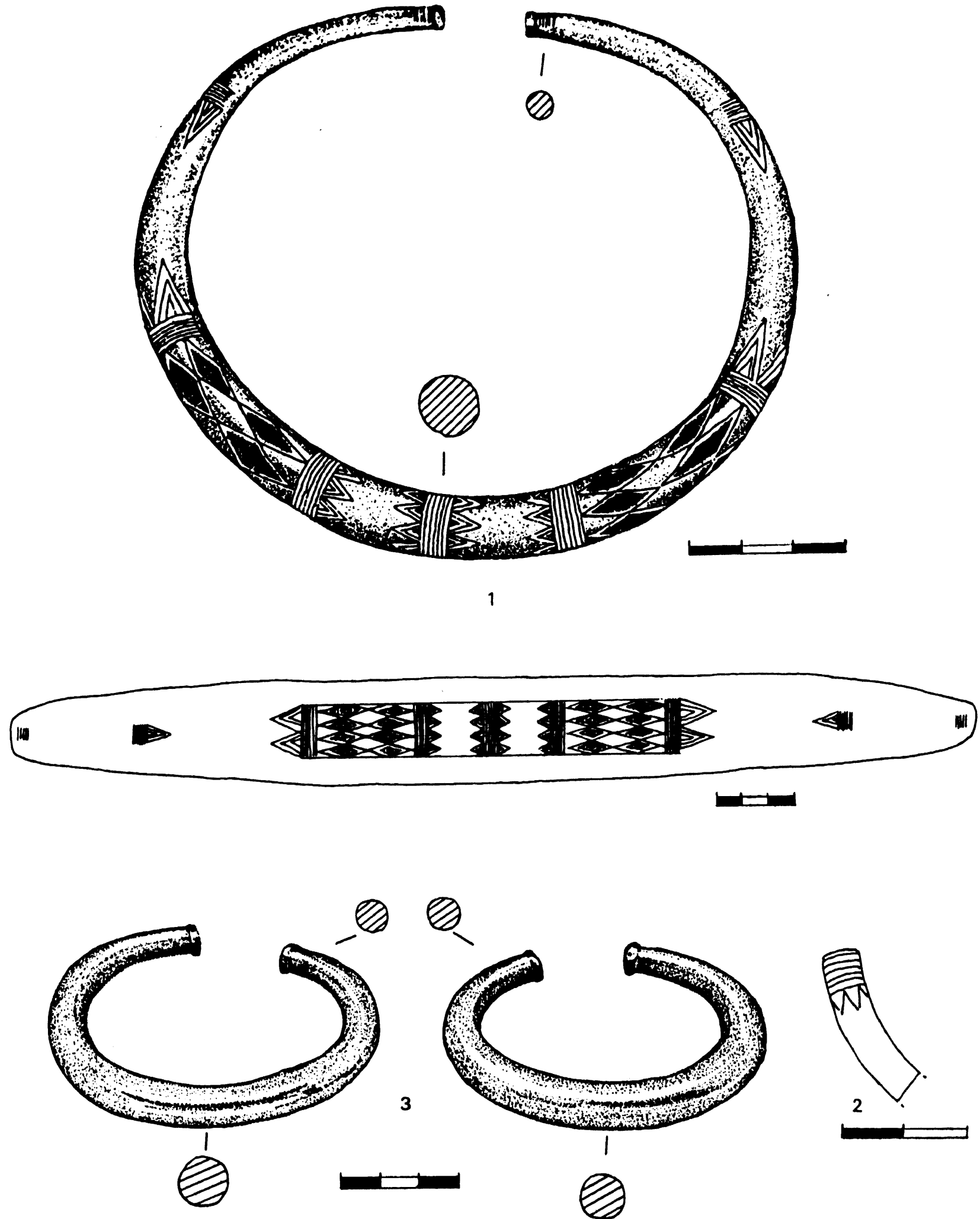

FIG. 1.-Torques y croquis de su decoración ( $\left.n^{\circ} 1\right)$, extremo de pulsera o brazalete decorado ( $\left.n^{\circ} 2\right)$ y brazaletes $n^{\circ}$ 3 y 4 ( $\left.n^{\circ} 3\right)$.

T. P., 1991, $\mathrm{n}^{2} 48$ 
un peso total de 1.174,60 gramos. Todos ellos fueron sometidos, en el Instituto de Conservación y Restauración de Bienes Culturales del Ministerio de Cultura, a análisis cuantitativo por la técnica no destructiva de fluorescencia de rayos X, espectómetro Revex 700, análisis de superficie en área grande. Los resultados los incluimos aqui por gentileza de D. Salvador Rovira.

El torques mide $12,7 \mathrm{~cm}$. de diámetro máximo, $1,2 \mathrm{cms}$. de grosor máximo y 0,5 de grosor minimo. Su peso es de 375,75 gramos. La composición que ofreció es la siguiente (Análisis PA 2008).

$\begin{array}{lrllll}\mathrm{Au} 90,35 \% & \mathrm{Ag} & 8,143 \% & \mathrm{Cu} & 1,144 \% & \mathrm{~m} \% \\ \mathrm{Sn} 0,861 \% & \mathrm{Fe} & 0,083 \% & \mathrm{Sb} & 0,0133 \%\end{array}$

La forma que ofrece es la de un aro amorcillado de sección circular, con grosor decreciente desde el centro, y extremos rematados por botones incipientes. Parece fundido a la cera perdida y en el interior se aprecia una grieta que bien pudo producirse al torcerse la pieza para conseguir la forma deseada.

Su decoración es a base de motivos geométricos incisos a buril, cuya composición y disposición parecen responder, en esencia, al mismo esquema que presenta Berzocana 1 (Almagro Gorbea, 1974: fig. 1 y 1977: fig. 6), algo simplificado. En la parte central y más gruesa se advierten siete líneas paralelas flanquedas por series de tres líneas inscritas en disposición de dientes de lobo. Hacia ambos lados, tras un espacio vacío, idénticas líneas en ángulos y otras siete paralelas enmarcando un ajedrezado de rombos lisos y otros incritos rellenos de reticulados que cubre la zona donde es más profusa la decoración. Nuevamente, tras un espacio vacio, líneas paralelas y en ángulos, esta vez las últimas sólo a un lado, a la altura del inicio de la mitad menos gruesa del aro y ya junto a los botones de los extremos únicamente líneas paralelas (Fig. 1, nº 1, Láms. Ib y IIa, b).

En cuanto a las pulseras o brazaletes, todas ellas son de oro macizo y sólo una presenta decoración. Las cuatro son de forma amorcillada y sección circular, con botones en los extremos (Fig. 1, nº 2-4; Lám. IIc).

La decorada, $\mathrm{n}^{\circ}$ 1, alcanza 201,78 gramos de peso, tiene un diámetro máximo de $8,3 \mathrm{cms}$, un grosor máximo de $1,1 \mathrm{cms}$. y el grosor mínimo es de $0,5 \mathrm{cms}$. Es en los extremos donde aparece una decoración de seis líneas paralelas incisas, precedidas de líneas en diente de lobo. Su análisis arrojó la siguiente composición (Análisis PA 2012):

$\begin{array}{lrlrllll}\mathrm{Au} & 90,69 \% & \mathrm{Ag} & 7,254 \% & \mathrm{Cu} & 1,561 \% & \mathrm{Ni} & 0,227 \% \\ \mathrm{Sn} & 0,114 \% & \mathrm{Fe} & 0,0128 \% & \mathrm{Sb} & 0,0131 \%\end{array}$

En esta pulsera se detectó un surco de extracción de metal hecho con lima fina de joyero (Fig. 1, $\mathrm{n}^{0}$ 2; Lám. IIc, d).

La no 2 pesa 198,8 gramos, el diámetro máximo es de $8,4 \mathrm{cms}$., el grosor máximo $1,05 \mathrm{cms}$. y el mínimo 0,6 cms. Su composición (Análisis ·PA 2010) quedó así:

$\begin{array}{lrlrllll}\mathrm{Au} & 92,07 \% & \mathrm{Ag} & 5,898 \% & \mathrm{Cu} & 1,536 \% & \mathrm{Ni} & 0,263 \% \\ \mathrm{Sn} & 0,118 \% & \mathrm{Fe} & 0,0101 \% & \mathrm{Sb} & 0,0126 \%\end{array}$

La pulsera $\mathrm{n}^{\circ} 3$ (Fig. 1, $\mathrm{n}^{0}$ 3) es de peso muy ligeramente inferior, 193,17 gramos, con 8,4 cms. de diámetro máximo, $1 \mathrm{~cm}$. de grosor máximo y $0,6 \mathrm{cms}$. del mínimo. El análisis (Análisis PA 2009) dio el resultado siguiente:

$\begin{array}{lrlrllll}\mathrm{Au} & 91,07 \% & & \mathrm{Ag} & 6,259 \% & \mathrm{Cu} & 1,525 \% \\ \mathrm{Sn} & 0,119 \% & \mathrm{Fe} & 0,0104 \% & \mathrm{Sb} & 0,0068 \%\end{array}$


La pulsera $\mathrm{n}^{9} 4$ (Fig. 1, $\mathrm{n}^{9}$ 3) es la de mayor peso 205, 32 gramos, con diámetro máximo de $8 \mathrm{~cm}$., un grosor máximo de $1,1 \mathrm{~cm}$. un mínimo de $0,7 \mathrm{~cm}$. Su análisis (PA 2011) proporcionó esta composición:

$\begin{array}{lrllllll}\text { Au } & 91,80 \% & \mathrm{Ag} & 6,347 \% & \mathrm{Cu} & 1,352 \% & \mathrm{Ni} & 0,169 \% \\ \mathrm{Sn} & 0,183 \% & \mathrm{Fe} & 0,0130 \% & \mathrm{Sb} & 0,0099 \%\end{array}$

En conjunto, las piezas presentan una gran homogeneidad en la composición del metal, en torno a los 22 quilates. Es también reseñable que, según el análisis realizado, la limpieza de la superficie del metal y de los surcos de la decoración hacen pensar en que fueron sometidas a un tratamiento de limpieza no abrasivo, tal vez baño detergente por ultrasonidos.

\section{COMENTARIO}

Es clara la vinculación de este tesorillo con la orfebrería tipo Sagrajas-Berzocana, de cuyo análisis y problemática se han ocupado diversos autores (Hawkes, 1971: 38 y ss.; Almagro Gorbea, 1974: 269 y ss. y 1977: 18 y ss.; Ruiz Gálvez, 1984: 399 y ss.; Coffyn, 1985: 236 y ss.) (1). Su probable procedencia extremeña reafirma la implantación en el occidente peninsular de este tipo de joyas emblemáticas, relacionadas, por un lado, con los influjos metalúrgicos atlánticos del Bronce final y también, en el aspecto social, con el surgimiento de élites o aristocracias locales que entroncan con la aparición de jefaturas y sociedades bien jerarquizadas (Ruiz Gálvez, 1988).

La pieza de mayor interés es el torques, al que hay que integrar dentro de la serie de torques de oro macizo decorados que caracterizan al citado tipo Sagrajas-Berzocana (Fig. 2). Es decir, los de Berzocana 1 y 2 (Callejo y Blanco, 1960: 250 y ss.), Sagrajas (Almagro Gorbea, 1974: 260 y ss.), el robado de Penela (Cartailhac, 1886; Reinach 1925: 124 y ss.), Evora (Reinach, 1925: 123 y ss.), Sintra (Leite de Vasconcelhos, 1895; Hawkes, 1971: 38 y ss.) y Baioes 1 y 2 (Kalb, 1978: 123; 1979, 582); fundamentalmente. Menos estrechas son ya las similitudes y paralelismos, a pesar de una relación cercana, con otras joyas del Bronce final occidental de sobra conocidas en su mayoría como el brazalete de Costa, los torques de Moura, Almoster, Serreces, etc. (Almagro Gorbea, 1974: 278 y 1977: 25 y ss.; Ruiz Gálvez, 1984: 399 y ss.; Coelho, 1985: 231 y ss).

Su forma de aro abierto lo emparenta más estrechamente con los de Berzocana y Baioes, sobre todo con Berzocana 1 y los de Baioes con los extremos rematados en botones. También la estructura de la pieza, de un sólo aro macizo, nos vuelve a remitir a los de Berzocana y Baioes, así como a los de Penela y Evora a pesar de que estos dos últimos tienen cierre. Otro aspecto que se ha valorado ha sido el del diámetro del grueso mayor, que resulta ser más del doble del menor, como ocurre con Berzocana 1 nuevamente y Sintra.

En cuanto a la decoración, ya hemos apuntado la similitud de la misma con la que presenta Berzocana 1, de manera especial la parte más gruesa del aro. También la tiene con la pieza 2 de Baioes y con Portel, Penela, Sagrajas e incluso no está alejada de la del collar de Sintra y el brazalete de Costa. Sin embargo, el esquema de "Valdeobispo" resulta ser uno de los más simples, situándose en este sentido tras el de Berzocana 2 y Baioes 1, más sencillos todavía y sin la presencia de rombos, rasgo éste que se ha valorado alguna vez como señal de antigüedad (Almagro Gorbea, 1974: 277). Resulta así una decoración menos elaborada que la que ofrecen la práctica totalidad de los torques tipo Sagrajas-Berzocana con excepción de Baioes 1 y Berzocana 2, éste último el ejemplar considerado tipológicamente más antiguo y de decoración más simple.

Por consiguiente, tanto la forma como la estructura y decoración relacionan de manera más

(1) Ruiz Gálvez (1984) cita frecuentemente un trabajo de Pingel: Goldfunde der Iberischen Halbinssel, entonces en preparación y que ignoramos si ya ha sido publicado.

$$
\text { T. P., } 1991, \mathrm{n}^{9} 48
$$




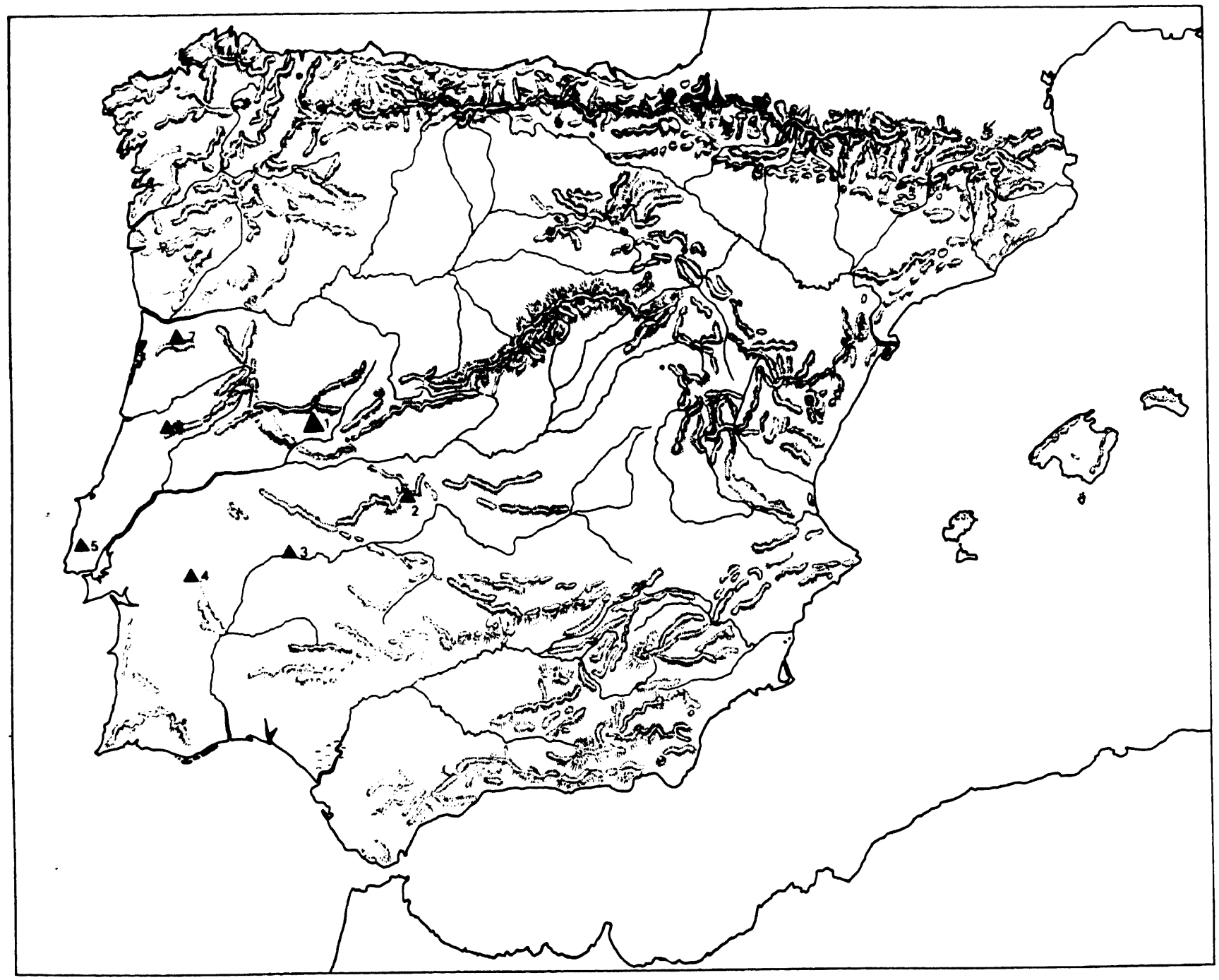

FIG. 2.-Dispersión de los torques más característicos de la orfebreria tipo Sagrajas-Berzocana: 1.- Valdeobispo; 2. Berzocana; 3.- Sagrajas; 4.- Evora; 5.- Sintra; 6.- Penela; 7.- Baioes.

estrecha la pieza de "Valdeobispo" con Berzocana 1 y las de Baioes, o sea con los torques de un aro abierto de extremidades acabadas en botones y decoración geométrica a base de combinaciónes más o menos simétricas de círculos, ángulos, rombos, etc. Unos motivos por cierto para los que algunos autores señalan correlaciones con las cerámicas portuguesas del Bronce final con decoración incisa (Coelho, 1986: 235; Iudice Gamito en prensa).

Dentro de la seriación generalmente aceptada para estos torques, habría que situar "Valdeobispo" a continuación de Berzocana 2 y Baioes 1 y con anterioridad a Berzocana 1 y tal vez Baioes 2 . La presencia de botones incipientes y de una decoración más elaborada que Berzocana 2 pueden señalar una mayor evolución, del mismo modo que ese poco desarrollo de los botones, más planos que cónicos, y el menor recargamiento ornamental pudieran indicar una mayor antigüedad, por lo menos estilística y formal, que Berzocana 1. En cualquier caso, Berzocana 1 y "Valdeobispo" presentan similitudes muy fuertes y no pueden estar muy alejados el uno del otro.

Por otra parte hay que apuntar que el torques de "Valdeobispo" es el que ofrece menor diámetro y menor peso de toda la serie hasta ahora conocida. Sus 375,57 gramos están muy lejos de los más de dos kilogramos que pesan los torques más complejos de Sagrajas y Evora, pero su 
peso es sensiblemente inferior también al de los de Baioes, que superan ambos el medio kilogramo, y al de Berzocana 1, su paralelo más cercano, que alcanza 950 gramos. Es incluso inferior al de Almoster que of rece 381,50 gramos. Este menor peso se ve compensado aunque mínimamente por su pequeño diámetro, 12,7 cms. frente a los 15 de Berzocana 1, 14,8 de Sagrajas o los 13,6 del menor de los de Baioes. Recordemos al respecto cómo Almagro Gorbea apuntó la posibilidad de que estas joyas estuvieran asociadas a las mujeres e incluso algunas destinadas a infantes (Almagro Gorbea, 1977: 61).

En cuanto a su composición metálica, no se aleja en exceso de la que ofrecen otras piezas áureas extremeñas del Bronce final (Hartmann, 1973, 1982), aunque no vamos a entrar en la discusión de la procedencia de los oros con que están hechos estos torques y adornos ya que los criterios son aún poco fiables (Ruiz Gálvez, 1985). Las impurezas de cobre y estaño no son altas y el porcentaje de plata es inferior al que ofrecen los torques de Berzocana (Hartmann 1982: 102).

Por último, hay que plantear la valoración cronológica del torques de "Valdeobispo", al respecto de lo cual tenemos que aludir a una cierta polémica a que están sujetas las fechas que se barajan para la orfebrería Sagrajas-Berzocana. El acuerdo es prácticamente unánime a la hora de señalar la falta de precedentes peninsulares para esta clase de joyas y se aceptan como prototipos piezas del Bronce medio y final de Bretaña, Isla Británicas y círculo nórdico. Tambien hay una cierta unanimidad a la hora de considerar que se trata de piezas fabricadas en la península en su mayor parte, como incorporaciones y sobre todo adaptaciones de otras foráneas (Ruiz Gálvez 1988: 344). Pero a la hora de fechar no todos los autores coinciden.

Así, Almagro Gorbea, a quien remite Coffyn, fecha este tipo de orfebrería entre los siglos XII/XI a. C. y el IX a. C. (Almagro Gorbea, 1977: 59 y 1985: 353), señalando mayor antigüedad para los ejemplares más sencillos - Berzocana 2- y mayor modernidad para aquéllos que cuentan con cierre y soldadura —Sagrajas, Sintra, etc.-. Ruiz Gálvez por su parte (Ruiz Gálvez, 1984: 399-400) recoge antiguas cronologías propuestas por Almagro Gorbea (1974: 274) y apunta fechas entre los siglos IX y VIII a. C., situando Berzocana 2, sin botones en los extremos en el siglo IX y Berzocana 1 y los de Baioes a mediados del VIII a. C., a los que seguirían los más complejos de Evora, Penela, Sagrajas y Sintra junto a otros tipos de torques. Al margen de los estilísticos, otro argumento que se esgrime para apoyar las cronologías más bajas es el de la fecha de C-14 proporcionada por la ocupación del castro de Ntra. Sra. de Guia de Baioes: $700 \pm 130$ (Kalb, 1974-77: 141), aunque ello lo que pudiera indicar es el momento de abandono de las piezas y el Castro (Coelho, 1986: 235).

Tomando como referencia el torques de Berzocana 1, el más cercano al de Valdeobispo, nos encontramos entre el siglo X propuesto por Almagro para esta pieza (Almagro Gorbea, 1977: 29) y la mitad del VIII a. C. de Ruiz Gálvez (1984: 400). Otros investigadores podrán discutir mejor que nosotros esta cuestión, pero parece claro que hay que considerar al torques de "Valdeobispo" como posterior a Berzocana 2 y Baioes 1 y algo anterior, o no muy alejado, a Berzocana 1 y Baioes 2, todos ellos abiertos y de un solo aro.

En cuanto a los brazaletes, es difícil hacer valoraciones intrínsecas de los mismos, por lo que debe prevalecer el criterio de asociación al torques. Con él además hay que relacionar las líneas y dientes de lobo del ejemplar decorado. Dentro de la región extremeña, tienen los brazaletes lisos un buen paralelo en el procedente de Monroy, también de forma amorcillada, sección circular y botones en los extremos y muy similar en cuanto a tamaño y peso: $7,9 \mathrm{cms}$. de diámetro máximo y 199,2 gramos de peso. Por otro lado, junto a los torques de Baioes se recogió un brazalete o pulsera muy similar a los presentados, aunque mucho más pesado: 385 gramos con lo que supera incluso al torques. Los que acompañaban al tesoro de Sagrajas presentan ya caracteres diferentes por ausencia de botón, aunque no deben estar cronológicamente muy distantes. De cualquier forma, brazaletes como los del tesorillo de "Valdeobispo" se conocen igualmente en el vecino Alentejo (Parreira y Vaz Pinto, 1980) y en general en todo el oeste peninsular (Schubart, 1975; Coelho, 1986).

Para concluir, una brevísima alusión al papel emblemático de piezas como estas dentro de las sociedades del Bronce final del occidente peninsular. En este sentido, y siguiendo lo recogido por Dalton (1975), Renfrew (1986) y Ruiz Gálvez (1988) entre otros, nos encontramos ante objetos que 
pueden considerarse de "valor primario" en tanto en cuanto se ponen a los de uso cotidiano y carecen de un verdadero valor práctico. Reafirman así la implantación de un lenguaje diferenciador de papeles y funciones sociales, asociado a personajes o grupos con capacidad de decisión y control, aunque su verdadero alcance dentro de las sociedades del momento lo desconozcamos todavia. No obstante, parece claro que quienes poseian joyas semejantes eran miembros de grupos que jugarian un destacado papel dentro de la dinámica de los sistemas comerciales y de intercambio de materias primas y elaboradas, al margen de que en concreto piezas de orfebrería como éstas pudieran tener un uso ceremonial exclusivo para las mujeres.

Por otro lado, el valor emblemático de estas joyas bien pudo ir cambiando con el tiempo, pero lo más seguro es que mantuviesen, a pesar de fluctuaciones, un valor intrínseco grande y de ahí la ocultación intencionada de muchos de estos tesorillos - hecho que no parece que responda a razones rituales- para preservarlos de eventualidades.

\section{BIBLIOGRAFIA}

Almagro Gorbea, M. (1974): "Los tesoros de Sagrajas y Berzocana y los torques de oro macizo del occidente peninsularm. Actas del III Congreso Nacional de Arqueologia: 259-282. Porto.

- (1977): El Bronce Final y el Periodo Orientalizante en Extremadura. Bibliotheca Praehistorica Hispana XIV, Madrid.

- (1986): «Bronce final y Edad del Hierro (La formación de las etnias y culturas prerromanas)». Historia de España 1. Prehistoria. Ed. Gredos. Madrid: 341-532.

Callejo, C. y Blanco, A. (1960): «El tesoro de Berzocana», Zephyrus, 11: 250-265. Salamanca.

Cartallhac, E. (1886): Les ages prehistoriques d'Espagne et Portugal.

Coelho Ferreira, A. (1986): A cultura castreja do noroeste de Portugal Paços de Ferreira.

CofFYN, A. (1985): Le Bronze final atlantique dans la Peninsule Iberique. Ed. Boccard. Paris.

DaltoN, G. (1975): «Karl Polanyi's analysis of long distance trade and his wider paradigm». In J. Sabloff and C. Lamberg-Karlowsky (eds).: Ancient civilization and trade. Alburquerque: 147-169.

HARTMAN, A. (1973): «Ergebnisse spektralanalyschen Untersuchungen Bronzezeitlichen Goldfunde der Iberischen Halbinselm. VIII Congrés du Union des Sciences Préhistoriques et Protohistoriques: 52-57. Belgrado.

- (1982): Prahistorische Goldfunde aus Europa II. Spektralanalyschen Untersuchungen und deren Auswertung. Studien zu den Anfängen der Metallurgie, 5. Berlín.

HAWKES, C. F. (1971): «The Sintra gold collar». The British Museum Quarterly, 39-50: 35. London.

Iudice Gamito, T. (en prensa): Paleoetnología de la Península Ibérica: Portugal: centro e sul.

KalB, Ph. (1974-77): «Uma data C-14 para o Bronce Atlánticon. O Arqueólogo Portugués, serie III, VII-IX: 141-144 Lisboa.

- (1978): «Senhora da Guia, Baioes. Die Aubgrabung 1977 auf einer hensiedlung der atlantischer bronzezeit in Portugal». Madrider Mitteilungen, 19: 112-139.

- (1979): "Contribución para el estudio del Bronce atlántico: Excavaciones en el castro "Señora da Guia" de Baioes (Concelho de Sao Pedro do Sul)». XV Congreso Nacional de Arqueología (Lugo, 1977): 583-591. Zaragoza.

Leite de Vasconcelhos, I. (1985): «Um torques de ouro». O Arqueólogo Portugués, I: Lisboa.

Parreira, R. y Vaz Pinto, C. (1980): Tesouros de Arqueologia Portuguesa no Museu Nacional de Arqueologia e Etnografía. Lisboa.

REINACH, S. (1925): «The Evora Gorget». The Antiquaries Journal, 5: 123-134.

RENFREW, C. (1986): «Varna and the emergence of wealth in prehistoric Europe». In A. Appadurai (ed.): The social life of things. Commodities in cultural perspective. C.U.P. Cambridge: 141-168.

Ruiz GálveZ, M. (1984): La península Ibérica y sus relaciones con el círculo cultural atlántico. Ed. Universidad Complutense. Madrid.

- (1985): «Recensión a A. Hartmann: Prähistorische Goldfunde aus Europa II (1982)». Boletín del Seminario de Arte y Arqueologia, LI: 528-532. Valladolid.

- (1988): "Oro y política. Alianzas comerciales y centros de poder en el Bronce final del occidente peninsular». Espacio, Tiempo y Forma. Serie I. Prehistoria. Homenaje al profesor Eduardo Ripoll Perelló. Madrid: 325-338.

SchubarT, H. (1975): Die Kultur der Bronzezeit in Südwesten der Iberischen Halbinsel. Madrider Forschungen 3. Berlín. 

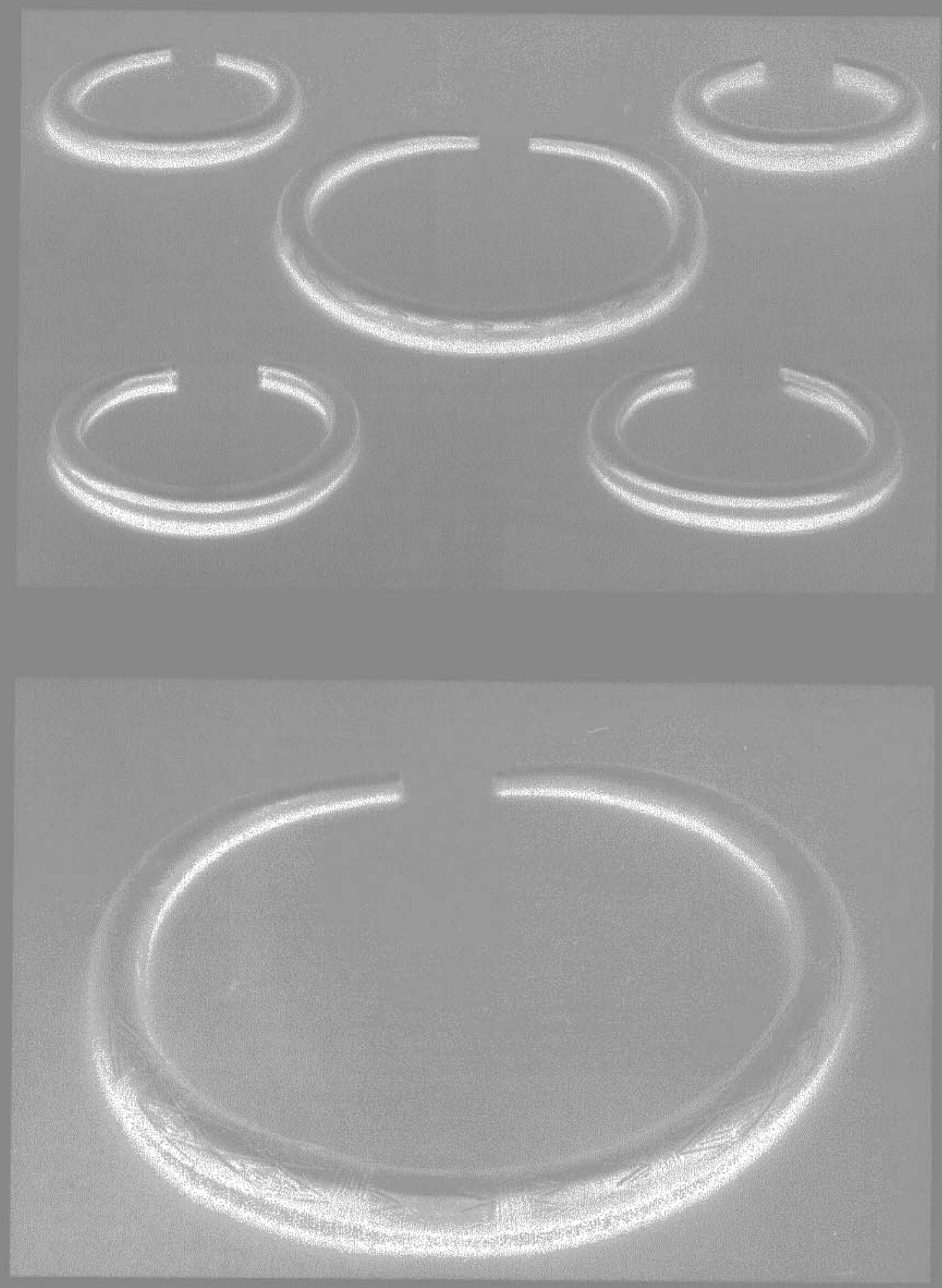

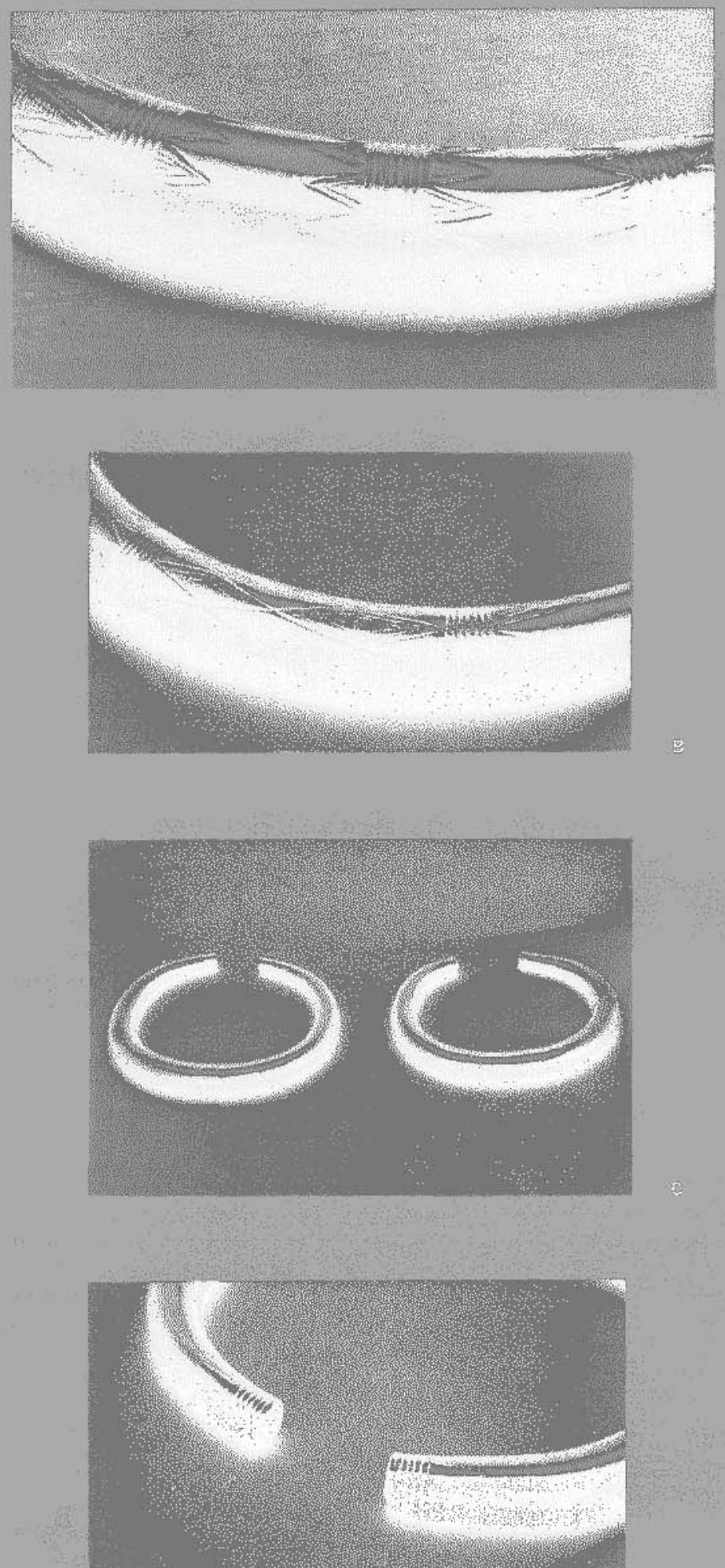